Research Article

\title{
Decision Making of Curriculum Attainment Degree for Engineering Geology Based on Fuzzy Set Theory
}

\author{
Binbin Yang $\mathbb{D}^{1},{ }^{1}$ Shuting Du $\mathbb{D},{ }^{1}$ Xiaoming Zhao $\mathbb{D},{ }^{1}$ Dongqi Tang $\left.\mathbb{D}\right)^{1}$ \\ and Changde Yang $\mathbb{D D}^{2}$ \\ ${ }^{1}$ School of Civil Engineering, Xuchang University, Xuchang 461000, Henan, China \\ ${ }^{2}$ School of Mining Engineering and Geology, Xinjiang Institute of Engineering, Urumqi 830023, \\ Xinjiang Uygur Autonomous Region, China
}

Correspondence should be addressed to Binbin Yang; ybb008008@hotmail.com

Received 1 November 2021; Accepted 15 November 2021; Published 26 November 2021

Academic Editor: bingxiang yuan

Copyright (C) 2021 Binbin Yang et al. This is an open access article distributed under the Creative Commons Attribution License, which permits unrestricted use, distribution, and reproduction in any medium, provided the original work is properly cited.

The study of engineering geology emphasizes the combination of theory and practice, and it highlights the comprehensive cultivation of curricular theory, curricular practice, and comprehensive skills. It is necessary to establish a set of effective methods to evaluate the achievement of training objectives to track, test, and improve the quality of curricular learning. In this paper, the principle of the fuzzy analytic hierarchy process based on entropy is used to construct an evaluation index system for the goal achievement degree of engineering geology courses, and the primary indicators mainly include knowledge, ability, and quality. Based on the actual situation, five curricular achievement levels are determined, that is, the comment set $V=\{$ very low, low, medium, high, very high\}. Relying on the engineering geology course resource database of Xuchang University, the course goal achievement degree is evaluated, and the results show that the engineering geology course achievement degree is high. This method is suitable not only for the evaluation of the achievement of curricular objectives but also for the evaluation of the achievement of engineering education graduation requirements. This method can also help us find different courses and teaching weak links supporting the index points, indicate the direction and provide support for teachers to continuously improve their teaching and management methods, and effectively promote a continuous improvement in teachers' teaching level and teaching quality.

\section{Introduction}

With the rapid development in the engineering construction, the relationship between engineering construction and the environment is becoming increasingly prominent, and the geological conditions of engineering construction are becoming increasingly complex $[1,2]$. Therefore, the new form has increasingly higher requirements for college students' innovative ability, which strengthens the demand for reform in colleges and universities with regard to the mode of diversified and personalized training of students' innovative ability, and it is necessary to cultivate engineering scientific and technological talent with innovative ability. The concept of outcome-based education (OBE) is student centered and student oriented, and it represents the mainstream direction of reform in the context of engineering education professional certification. Engineering education professional certification advocates the concept of OBE throughout the whole process of talent training. It is an internationally accepted engineering education quality assurance system. Many domestic colleges and universities use this concept and method in specialty construction and talent training [3]. The gradual application of modern information technologies such as the Internet and big data in education and teaching has triggered changes in traditional majors and disciplines such as civil engineering and forced colleges and universities to reconstruct the talent training system for traditional industries. Especially in recent years, due to the impact of global climate change, the frequency and intensity of extreme climate events have increased significantly, 
affecting geological bodies in various ways and inducing a series of engineering geological disasters, such as subgrade collapses and house cracking [4-7].

Resource database construction is an important means of realizing educational informatization and a learning society. It relies on network technology, teaching resources, and big data to improve educational modernization and informatization. A Hadoop-based cloud computing platform is built for big data analysis and application course resource management, improves resource utilization and sharing, and provides better services for teachers, students, and employees [8]. The number of web-based continuing education courses has increased significantly, but educators know little about the factors affecting the use of resources in online courses or the relationship between resource use and educational outcomes. The rapid development of online education, knowledge sharing, big data, and artificial intelligence technology has brought innovation to education [9]. Gueudet and Poisard discussed the design and use of curriculum resources brought by the use of the literature method in teaching methods, and they proposed this theoretical method and the related "reflective investigation" method [10]. By referring to the latest studies on digital curriculum resources (DCRs), this paper discusses the design and use (by teachers and students) of digital curricula and electronic textbooks in mathematics education $[11,12]$. With the rapid development of network technology and the sharing and promotion of intelligent vocational education platforms, the role of the resource database in "Internet + education" is particularly important [13]. An online course system platform has the function of promoting active, collaborative, interactive, research-based, and autonomous learning and is an important way to form an open, efficient, and convenient new teaching mode $[14,15]$. Taking the creation of high-quality resources, network teaching, improved teaching effects, enriched teaching means, and improved teaching effects as the core realizes threedimensional teaching involving the rapid upload, retrieval, and archiving of resources. A perfect online course system is the basis and guarantee for all-round information-based teaching and for improving the level of school education information $[16,17]$.

The fuzzy comprehensive evaluation method is a comprehensive bid evaluation method based on fuzzy mathematics. It is characterized by strong systematicness and clear and reliable results. It can better solve fuzzy and difficult-toquantify problems. It is suitable for quantitative analysis and qualitative research [18]. Chen et al. combined a fuzzy analytic hierarchy process with a fuzzy comprehensive evaluation method and proposed a new teaching performance evaluation framework. The application of this framework can make the evaluation results more scientific, accurate, and objective. It is hoped that this work can help university managers improve the level of educational quality [19]. Liu and Chen constructed a fuzzy matrix through membership determination on the basis of five first-level objectives and second-level corresponding objectives. The matrix can objectively and fairly evaluate students' learning effect, stimulate students' enthusiasm for autonomous learning, and help them build their confidence in employment challenges with an optimistic and positive attitude [20]. The fuzzy comprehensive evaluation method is a method based on fuzzy mathematics. By constructing the weight, the index can be evaluated objectively and scientifically. Using a fuzzy comprehensive evaluation method can help teachers improve their teaching quality in a timely manner, change the time control of teaching quality into process control, and change static management into dynamic management [21-23].

The study of engineering geology courses emphasizes the combination of theory and practice and highlights the comprehensive cultivation of curricular theory, curricular practice, and comprehensive skills. It is necessary to establish a set of effective methods to evaluate the achievement of training objectives to track, test, and improve the quality of curricular learning. Taking the civil engineering students of Xuchang University as the evaluation object, based on the principle of the fuzzy analytic hierarchy process, this paper constructs an evaluation index system for the engineering geology curricular goal achievement degree, carries out a reasonable weight distribution, inspection, and optimization, formulates the evaluation grade evaluation standard, and realizes the goal of curricular achievement degree evaluation considering a variety of evaluation data. It can provide a reference for graduation achievement evaluation for engineering education certification.

\section{Methodology}

The objects can be evaluated by the fuzzy comprehensive evaluation method based on multiple factors according to the fuzzy relationship synthesis. There is a unique evaluation value for the evaluated object in the fuzzy comprehensive evaluation method, which is not affected by the set of evaluation objects. Each index can be evaluated by the fuzzy comprehensive evaluation method. The weight of each influence index can quantitatively calculate according to the historical statistical data, and the object can be evaluated quantitatively [24]. A fuzzy comprehensive evaluation method was used in this paper to calculate and evaluate the achievement degree of engineering geology courses based on the course resource database. The calculation and evaluation steps of this method are as shown in Figure 1.

\subsection{Determining the Evaluation Factor Set and Index System.} The factor set is a set composed of various factors affecting the evaluation object as elements, set as $U=\left\{u_{1}, u_{2}, \ldots, u_{n}\right\}$. $U$ is the $\mathrm{N}$ factor describing the evaluation object, namely, the evaluation index [25]. Based on the role of engineering geology in the curricular system and in combination with the graduation requirements, the curricular objectives are divided into three parts to support the first-class indicators of the graduation requirements, as shown in Table 1 and Figure 2.

\subsection{Determining the Comment Set of Comprehensive}

Evaluation. The comment set is a set of possible results for various indicators, set as $\mathrm{V}=\left\{v_{1}, v_{2}, v_{3}, \ldots, v_{\mathrm{n}}\right\}$. V refers to $\mathrm{N}$ kinds of decisions describing the state of each factor, and $\mathrm{N}$ refers to the number of comments, which is generally 


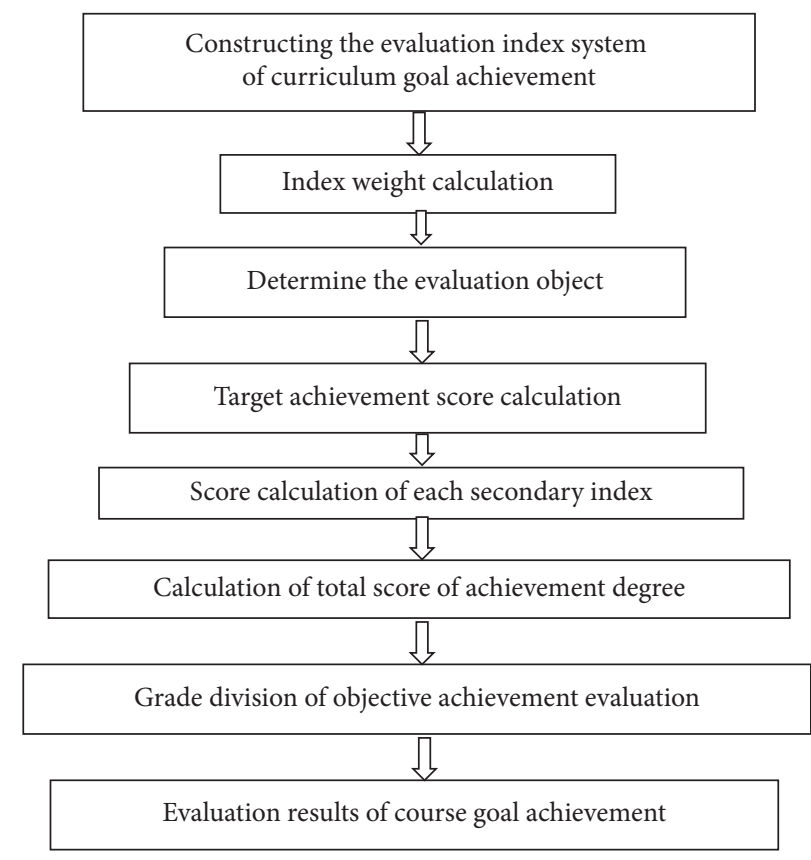

FIgURE 1: Course goal achievement evaluation process.

TABLE 1: Correspondence between the course objectives and graduation requirements.

Graduation requirement index

Knowledge objectives

Capability objectives

Quality objectives

\section{Course objective}

To master the basic concepts, principles, and advanced methods of engineering geology to be able to solve complex engineering geological problems in the life cycle of civil engineering

To improve the ability of engineering practice and innovation, the ability to deal with geological disasters in engineering, and the ability to engage in independent learning and teamwork

To have good scientific and cultural literacy, ideological, and political literacy and a sense of social responsibility and professional ethics, to have engineer thinking and a craftsman's spirit, and to have the correct outlook on life, values, and world view

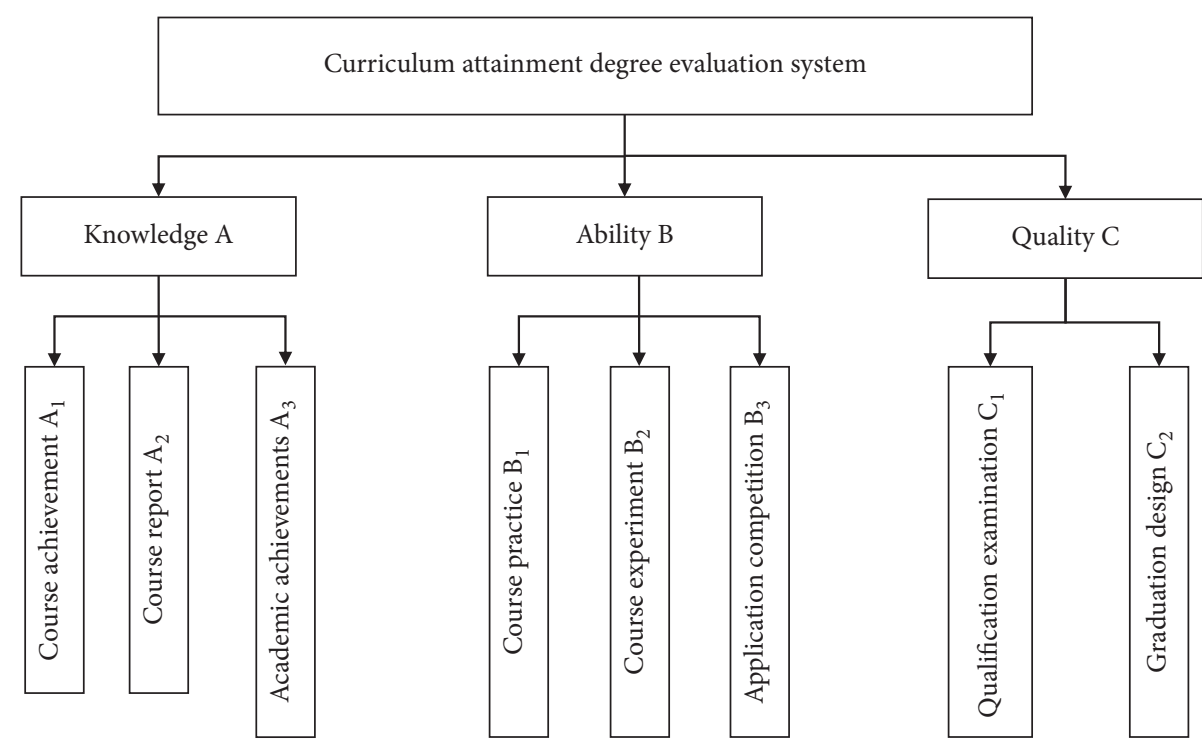

Figure 2: Curricular goal achievement index system. 
divided into 3-5 levels [26]. Based on the actual situation, this paper determines five curricular achievement levels, that is, the comment set $\mathrm{V}=$ \{very low, low, medium, high, very high\} [27, 28].

2.3. Determining the Factor Weight Vector. In evaluation, the weight is a measure of the relative importance of factors. If the weight cannot be determined objectively, the evaluation results may be seriously distorted and even cause judges to make the wrong judgment. In this paper, the weight of the evaluation factors is quantitatively determined by the entropy method through statistical empirical data. The weight set of each factor or the weight vector is represented by $A=$ $\left(a_{1}, a_{2}, a_{3}, \ldots, a_{i}\right)$, where $a_{i}>0$ and $\sum a_{\mathrm{i}}=1$, which reflects a trade-off of various factors.

In multifactor evaluation, different weights are taken because different factors have different practical roles in evaluation. In this paper, the weight of each factor is calculated by statistical data on a course resource (Table 2) and the entropy method model. In information theory, entropy is a measure of uncertainty. The greater the amount of information is, the smaller the uncertainty and the smaller the entropy. The smaller the amount of information is, the greater the uncertainty and the greater the entropy [29]. Based on the characteristics of entropy, an event's randomness and disorder degree can be judged by calculating the entropy, and the dispersion degree of an index can also be judged by the entropy. The greater the dispersion degree of the index is, the greater the impact of the index on comprehensive evaluation $[30,31]$. The specific calculation is as follows.

Calculate the proportion of this index under the influence of factor $j$.

$$
p_{i j}=\frac{x_{i j}}{\sum_{i=1}^{n} x_{i j}}, \quad(i=1,2 \ldots n ; j=1,2 \ldots m) .
$$

Calculate the entropy of factor $j$.

$$
\begin{aligned}
& e_{j}=-k \sum_{i=1}^{n} p_{i j} \ln \left(p_{i j}\right), \quad k>0, \\
& k=\frac{1}{\ln (n)}, \quad e_{j} \geq 0 .
\end{aligned}
$$

Calculate the difference coefficient of index $j$. For the $j$-th index, the greater the difference of index $\mathrm{X}_{i j}$ is, the greater the impact on safe mining and the smaller the entropy.

Define the difference factor:

$$
\begin{aligned}
g_{j} & =\frac{1-e_{j}}{m-E_{e}}, \\
E_{e} & =\sum_{j=1}^{m} e_{j}, \quad 0 \leq g_{j} \leq 1, \\
\sum_{j=1}^{m} g_{j} & =1 .
\end{aligned}
$$

The weight results are obtained as follows:
TABLE 2: Weights of the secondary evaluation indexes.

\begin{tabular}{lcc}
\hline Primary index & Secondary index & Weight \\
\hline \multirow{3}{*}{ Knowledge } & Course achievements & 0.33 \\
& Course reports & 0.35 \\
& Academic achievements & 0.32 \\
\hline \multirow{3}{*}{ Ability } & Course practice & 0.25 \\
& Course experiments & 0.41 \\
Quality & Application competitions & 0.34 \\
\hline \multirow{2}{*}{ Qualification examinations } & 0.48 \\
& Graduation design & 0.52 \\
\hline & & \\
& $g_{j}=\frac{}{\sum_{j=1}^{m} g_{j}}(1 \leq j \leq m)$.
\end{tabular}

\subsection{The Single Factor Fuzzy Evaluation Is Carried Out, and the} Evaluation Matrix Is Constructed. First, make a single factor evaluation of the single factor $\mathrm{U}_{i}(i=1,2,3, \ldots, \mathrm{n})$ in the factor set. From the membership of the factor $U_{i}$ to the evaluation grade $\mathrm{V}_{j}$ as $\mathrm{R}_{i j}$, we can obtain the single factor evaluation set of the $i$-th factor $\mathrm{U}_{i}$, and $R_{i}=\left(R_{i 1}, R_{i 2}, \ldots, \mathrm{R}_{i \mathrm{n}}\right)$. In this process, the determination of membership is very important. This paper uses the questionnaire method combined with the actual learning situation of students to obtain the membership of the determined factors. Finally, a general evaluation matrix $R$ is constructed from the evaluation set of $\mathrm{N}$ factors. That is, each evaluated object determines the fuzzy relationship matrix $R$ from $\mathrm{U}$ to $\mathrm{V}$.

2.5. Fuzzy Synthesis and Judgment. A fuzzy subset B of evaluation set $\mathrm{V}$ is introduced, which is called the evaluation set, that is, $B=\left(b_{1}, b_{2}, b_{3}, \ldots, \mathrm{b}_{n}\right)$. Let $B=A^{*} \mathrm{R}$ ( ${ }^{*}$ is the operator symbol), which is the fuzzy comprehensive evaluation model. To make the evaluation results clear, the information concentration principle is adopted and scored based on the percentage system. The information concentration formula is

$$
S=B * C^{T} .
$$

Among them, $C^{\mathrm{T}}$ is the grade of the corresponding factors. Based on the final score $S$, the achievement degree of the course can be judged by comparing and dividing the interval (Table 3).

\section{Case Study}

Taking the civil engineering students (64 in total) enrolled in the engineering geology course at Xuchang University as an example, the required data are obtained from the online course system. Civil engineering is the earliest undergraduate major in the School of Civil Engineering of Xuchang University which located in Xuchang City, Henan Province, in China. The students trained by civil engineering have solid theoretical foundation and professional knowledge, good scientific and cultural literacy, sense of social responsibility and professional ethics, and practical ability and innovation 
TABLE 3: The assessment grade of achievement.

\begin{tabular}{lccccc}
\hline $\begin{array}{l}\text { Achievement } \\
\text { rating }\end{array}$ & $\begin{array}{l}\text { Very } \\
\text { low }\end{array}$ & Low & Medium & High & $\begin{array}{l}\text { Very } \\
\text { high }\end{array}$ \\
\hline $\begin{array}{l}\text { Achievement } \\
\text { score }\end{array}$ & $<40$ & $40-59$ & $60-69$ & $70-89$ & $90-100$ \\
\hline
\end{tabular}

ability to independently solve complex engineering problems in the field of civil engineering, as well as international vision and team cooperation ability.

The evaluation indicators are divided into two levels. The first-level indicators mainly include knowledge, ability, and quality. The knowledge indicators are mainly controlled by the second-level indicators: course achievements, course reports, and academic achievements. The relationship matrix is shown in the following equation:

$$
R_{A}=\left(\begin{array}{ccccc}
0 & 0.12 & 0.27 & 0.29 & 0.32 \\
0 & 0 & 0 & 0.29 & 0.71 \\
0.05 & 0.13 & 0.45 & 0.33 & 0.04
\end{array}\right)
$$

Course practice, course experiments, and application competitions are the main factors affecting the ability index. The constructed relationship matrix is shown in the following equation:

$$
R_{B}=\left(\begin{array}{ccccc}
0 & 0 & 0 & 0.27 & 0.73 \\
0 & 0.14 & 0.33 & 0.42 & 0.11 \\
0 & 0.21 & 0.25 & 0.33 & 0.21
\end{array}\right) .
$$

The main factors affecting the quality indicators are quality examinations and graduation design, and the constructed relationship matrix is shown in the following equation:

$$
R_{C}=\left(\begin{array}{lllll}
0.002 & 0.212 & 0.415 & 0.274 & 0.097 \\
0.001 & 0.245 & 0.365 & 0.285 & 0.104
\end{array}\right) .
$$

Based on the information entropy model, the weights of the secondary indicators are calculated, and the weight values of the secondary indicators are obtained, as shown in Table 2. The weight vectors of each secondary index are $\left(w_{A 1}\right.$, $\left.w_{A 2}, w_{A 3}\right)=(0.33,0.35,0.32),\left(w_{B 1}, w_{B 2}, w_{B 3}\right)=(0.25,0.41$, $0.34)$, and $\left(w_{C 1}, w_{C 2}\right)=(0.48,0.52)$. Through the synthesis operation of the membership matrix, the comprehensive evaluation matrix of the primary indexes is obtained, as shown in the following equation:

$$
R=\left(\begin{array}{ccccc}
0.016 & 0.0812 & 0.2331 & 0.3028 & 0.3669 \\
0 & 0.1288 & 0.2203 & 0.3519 & 0.299 \\
0.00148 & 0.22916 & 0.389 & 0.27972 & 0.10064
\end{array}\right)
$$

The setting of the evaluation target value is related to the overall teaching level of the school, reflecting the strictness of the requirements for students. Considering that the school is still dominated by the school year system, the setting of the evaluation target value is based on whether students pass the assessment of corresponding links. The weight vector of the first-level evaluation index is $(0.34,0.33,0.33)$. Through the evaluation set $B=\left(b_{1}, b_{2}, b_{3}, b_{4}, b_{5}\right)$, where $B=A^{*} \mathrm{R}\left({ }^{*}\right.$ is the operator symbol), obtain $B=(0.005928,0.1457348$, $0.280323,0.3113866,0.2566272) . B$ is the comprehensive evaluation result matrix of the evaluation achievement degree, which is the membership degree of different quality levels. $b_{1}, b_{2}, b_{3}, b_{4}$, and $b_{5}$ represent low, low, medium, high, and high membership, respectively. $A$ is the weight matrix of the evaluation factor, and $R$ is the evaluation matrix. Based on the principle of information concentration, $s=74.25$ is obtained from equation (5). According to Table 3, the degree of achievement of the engineering geology course is high.

\section{Conclusion}

Based on the principle of the fuzzy analytic hierarchy process based on entropy, this paper constructs an evaluation index system for the engineering geology curricular goal achievement degree. Relying on the engineering geology curriculum resource database of Xuchang University, this paper evaluates the curricular goal achievement degree and obtains good application results. This method is applicable not only to the evaluation of the degree of achievement of curricular objectives but also to the evaluation of the achievement of graduation requirements for engineering education certification, and it can also help us find different courses and teaching weak links supporting the index points, indicate the direction and provide support for teachers to continuously improve their teaching and management methods, and effectively promote a continuous improvement in teachers' teaching level and teaching quality. Based on the principle of information concentration, $s=74.25$, the degree of achievement of the engineering geology course is high.

Fuzzy mathematics and information entropy theory essentially solve the problems of unsystematic index selection and the difficult combination of qualitative and quantitative evaluation in the process of curricular goal achievement evaluation. The example application shows that the curricular goal achievement evaluation system constructed in this paper can be used as an effective reference method for university decision-making. Through the evaluation process of the degree of achievement of the curricular objectives in this paper, we see that to achieve the curricular objectives, we should pay attention to the details of the overall curricular teaching quality control and curricular organization to a great extent. We should not only consolidate the accumulation of basic theoretical knowledge and skills but also strengthen the cultivation of practice, training, and competition ability and encourage innovation.

\section{Data Availability}

The datasets generated during the current study are available from the corresponding author on reasonable request.

\section{Conflicts of Interest}

The authors declare no conflicts of interest in the submission of this manuscript, and the manuscript is approved by all the authors for publication. 


\section{Acknowledgments}

This work was financially supported by the Pedagogical Research and Practice Project of Xuchang University under Grant No. XCU2021-YB-047 and No. XCU2021-ZB-003.

\section{References}

[1] B. Yuan, Z. Li, Z. Zhao, H. Ni, Z. Su, and Z. Li, "Experimental study of displacement field of layered soils surrounding laterally loaded pile based on transparent soil," Journal of Soils and Sediments, vol. 21, no. 9, pp. 3072-3083, 2021.

[2] J. He, Y. Xue, J. Xu, D. Zhang, and S. Zhang, "Whole-process monitoring of sinkhole collapse based on distributed optical fiber strain-vibration joint system and its case study in railway subgrade," Optical Fiber Technology, vol. 60, Article ID 102380, 2020.

[3] R. M. Harden, "Developments in outcome-based education," Medical Teacher, vol. 24, no. 2, pp. 117-120, 2002.

[4] T. C. Su, "Assessment of cracking widths in a concrete wall based on tir radiances of cracking," Sensors, vol. 20, no. 17, 2020.

[5] C. A. Pederson, P. M. Santi, and D. R. Pyles, "Relating the compensational stacking of debris-flow fans to characteristics of their underlying stratigraphy: implications for geologic hazard assessment and mitigation," Geomorphology, vol. 248, pp. 47-56, 2015.

[6] B. Y. Yuan, Z. H. Li, Z. L. Su, Q. Z. Luo, M. J. Chen, and Z. Q. Zhao, "Sensitivity of multistage fill slope based on finite element model," Advances in Civil Engineering, vol. 2021, Article ID 6622936, 13 pages, 2021.

[7] B. Y. Yuan, Z. H. Li, Y. Chen et al., "Mechanical and microstructural properties of recycling granite residual soil reinforced with glass fiber and liquid-modified polyvinyl alcohol polymer - sciencedirect," Chemosphere, vol. 268, Article ID 131652, 2021.

[8] Y. Zhao and H. Liu, "Cloud curriculum resource management platform based on Hadoop," Measurement and Control, vol. 53, no. 9-10, pp. 1782-1790, 2020.

[9] C. R. Woods and K. J. Kemper, "Curriculum resource use and relationships with educational outcomes in an online curriculum," Academic Medicine, vol. 84, no. 9, pp. 1250-1258, 2009.

[10] G. Gueudet and C. Poisard, "Design and use of curriculum resources for teachers and teacher educators: example of the Chinese abacus at primary school," International Journal of Educational Research, vol. 93, pp. 68-78, 2019.

[11] B. Pepin, J. Choppin, K. Ruthven, and N. Sinclair, "Digital curriculum resources in mathematics education: foundations for change," ZDM, vol. 49, no. 5, pp. 645-661, 2017.

[12] X. Y. Zhang, A. M. Holbrook, L. Nguyen et al., "Evaluation of online clinical pharmacology curriculum resources for medical students," British Journal of Clinical Pharmacology, vol. 85, no. 11, pp. 2599-2604, 2019.

[13] A. M. Bodzin, Q. Fu, V. Kulo, and T. Peffer, "Examining the effect of enactment of a geospatial curriculum on students' geospatial thinking and reasoning," Journal of Science Education and Technology, vol. 23, no. 4, pp. 562-574, 2014.

[14] B. Y. Chen, D. E. Kern, R. M. Kearns, P. A. Thomas, M. T. Hughes, and S. Tackett, "From modules to MOOCs," Academic Medicine, vol. 94, no. 5, pp. 678-685, 2019.

[15] S. Rezat, L. Fan, and B. Pepin, "Mathematics textbooks and curriculum resources as instruments for change," ZDM Mathematics Education, vol. 53, no. 6, pp. 1189-1206, 2021.
[16] Z. Karimi, T. Ashktorab, E. Mohammadi, H. Abedi, and K. Zarea, "Resources of learning through hidden curriculum: Iranian nursing students' perspective," Journal of Education and Health Promotion, vol. 4, p. 57, 2015.

[17] M. Misfeldt, A. L. Tamborg, J. Dreyøe, and B. B. Allsopp, "Tools, rules and teachers: the relationship between curriculum standards and resource systems when teaching mathematics," International Journal of Educational Research, vol. 94, pp. 122-133, 2019.

[18] S. D. Xian, "A new fuzzy comprehensive evaluation model based on the support vector machine," Fuzzy Information And Engineering, vol. 2, no. 1, pp. 75-86, 2010.

[19] J. F. Chen, H.-N. Hsieh, and Q. H. Do, "Evaluating teaching performance based on fuzzy AHP and comprehensive evaluation approach," Applied Soft Computing, vol. 28, pp. 100108, 2015

[20] S. Liu, P. Chen, and P. Chen, "Research on fuzzy comprehensive evaluation in practice teaching assessment of computer majors," International Journal of Modern Education and Computer Science, vol. 7, no. 11, pp. 12-19, 2015.

[21] Y. Jiang and Y. Wang, "Evaluation of teaching quality of public physical education in colleges based on the fuzzy evaluation theory," Journal of Computational and Theoretical Nanoscience, vol. 13, no. 12, pp. 9848-9851, 2016.

[22] S. W. Sun and Y. W. Zhai, "The application of fuzzy comprehensive evaluation model in the teaching evaluation system," Applied Mechanics and Materials, vol. 303-306, pp. 1452-1455, 2013.

[23] F. Jiang, Q. Zheng, and W. Shi, "The applied research of fuzzy comprehensive evaluation on talent training mode of safety engineering," Procedia Engineering, vol. 43, pp. 425-430, 2012.

[24] S. Feng and L. D. Xu, "Decision support for fuzzy comprehensive evaluation of urban development," Fuzzy Sets and Systems, vol. 105, no. 1, pp. 1-12, 1999.

[25] Y.-W. Du, S.-S. Wang, and Y.-M. Wang, "Group fuzzy comprehensive evaluation method under ignorance," Expert Systems with Applications, vol. 126, pp. 92-111, 2019.

[26] L.-j. Li and L.-t. Shen, "An improved multilevel fuzzy comprehensive evaluation algorithm for security performance," The Journal of china Universities of Posts and Telecommunications, vol. 13, no. 4, pp. 48-53, 2006.

[27] H. Zhang, X. He, and H. Mitri, "Fuzzy comprehensive evaluation of virtual reality mine safety training system," Safety Science, vol. 120, pp. 341-351, 2019.

[28] K.-L. Wen, "A Matlab toolbox for grey clustering and fuzzy comprehensive evaluation," Advances in Engineering Software, vol. 39, no. 2, pp. 137-145, 2008.

[29] Q. S. Zhang and S. Y. Jiang, "A note on information entropy measures for vague sets and its applications," Information Sciences, vol. 178, no. 21, pp. 4184-4191, 2008.

[30] D. L. Mon, C.-H. Cheng, and J. C. Lin, "Evaluating weapon system using fuzzy analytic hierarchy process based on entropy weight," Fuzzy Sets and Systems, vol. 62, no. 2, pp. 127-134, 1994.

[31] M. Song, Q. Zhu, J. Peng, and E. D. R. G. Santibanez, "Improving the evaluation of cross efficiencies: a method based on Shannon entropy weight," Computers \& Industrial Engineering, vol. 112, pp. 99-106, 2017. 